\section{Associação entre exercício físico e propríocepcão em idosos: uma revisão sistemática}

\author{
Relation between physical exercise and \\ proprioception in elderly: a systematic \\ review
}

\author{
Karini Borges dos Santos \\ Araceli Goedert ${ }^{1}$ \\ Paulo C. B. Bento \\ André L. F. Rodacki ${ }^{1}$
}

\section{RESUMO}

O comprometimento proprioceptivo decorrente do envelhecimento aumenta o risco de queda entre os idosos. Exercício físico pode atenuar o processo de declínio da sensação consciente da posição do corpo. Esta revisão sistemática teve como objetivo associar estudos que relacionam o exercício físico com a propriocepção em idosos. Realizou-se uma busca em três bases de dados eletrônicas (Pubmed/Mediline, Lilacs e Scopus) nos últimos 10 anos com a seguinte associação de descritores: (elderly OR aged) AND (proprioception OR position sense) AND exercise. A busca gerou 271 artigos e após aplicação de critérios de inclusão e exclusão, nove estudos foram selecionados para compor a revisão sistemática. Todos os artigos mostraram uma associação entre propriocepção e algum exercício físico. Tai chi chuan e Sessões de treinamento multifatorial foram as modalidades que apareceram com maior frequência entre os estudos (33 e 56\% respectivamente), outras atividades compreenderam golfe, natação, corrida, fortalecimento muscular, flexibilidade, caminhada, exercícios de agilidade, equilíbrio, postura e de propriocepção. Tai chi chuan, séries multifatoriais, golfe e exercícios de propriocepção demonstraram ser as estratégias mais espressivas na melhoria da propriocepção entre os idosos.

\section{PALAVRAS-CHAVE}

Envelhecimento; Propriocepção; Atividade física.

\begin{abstract}
As a consequence of aging the proprioceptive system is compromised, increasing the risk of falls among the elderly. Exercise may attenuate the decline of conscious sensation of body position. This systematic review aimed to identify and describe some studies linking exercise with proprioception in the elderly. A search was conducted in three databases (PubMed/Medline, Lilacs and Scopus) for the last 10 years, with the following combination of descriptors: ((elderly OR aged) AND (sense position OR proprioception) AND exercise). The search generated 271 articles, and after the application of inclusion and exclusion criteria, 9 studies were selected to compose this review. All studies showed an association between proprioception and some kind of exercise. Tai chi chuan and multifactorial training sessions appeared more frequently among these articles, while other related activities were golf, swimming, jogging, muscle strengthening, flexibility, walking, agility, balance, posture and proprioception exercise. Tai chi chuan, multifactorial series, golf and proprioception exercises proved to be the most beneficial strategies in improving proprioception.
\end{abstract}

\section{KEYWORDS}

Elderly; Proprioception; Physical Activity .
Rev Bras Ativ Fís Saúde p. 17-25 DOI

http://dx.doi.org/10.12820/rbafs.v.20n1p17

1 Universidade Federal do Paraná. R. Coração de Maria, 92 - Jardim Bontânico - Setor de Ciências Biológicas - Curitiba, Paraná. 


\section{INTRODUÇÃO}

O aumento substancial na expectativa de vida e nos avanços com cuidados relacionados à saúde no século XXI tem induzido grande interesse na promoção de um envelhecimento mais saudável ${ }^{1}$. Entre os principais problemas de saúde que afetam pessoas a partir dos sessenta anos de idade estão às quedas acidentais e suas implicações em morbidades, redução da funcionalidade, perda da independência e em casos extremos, a morte ${ }^{2}$. Vários fatores podem contribuir para as quedas entre idosos, todavia, os comprometimentos sensoriais decorrentes do envelhecimento têm sido apontados como um dos elementos centrais que podem resultar em déficits no controle postural e no equilíbrio ${ }^{3-5}$.

A propriocepção é definida como o conjunto de informações somatossensoriais enviadas constantemente ao sistema nervoso central por um conjunto de receptores. Os receptores são estruturas localizadas nos músculos, ligamentos e cápsulas articulares e são os receptores primários das alterações de posicionamento corporal. Outros receptores são localizados mais superficialmente e permitem o reconhecimento de estímulos nocivos, como dor, calor e pressão excessiva ${ }^{6,7}$. O conjunto de informações sensoriais influencia o controle postural durante a postura estática e dinâmica ${ }^{8-10}$.

Exercícios físicos têm sido empregados como uma estratégia benéfica para atenuar o declínio da propriocepção decorrente do envelhecimento ${ }^{11}$. De fato, alguns estudos apontam uma relação entre ganhos de força muscular, equilíbrio e funcionalidade com melhorias da propriocepção ${ }^{12}$. Todavia, diferentes formas de atividades físicas têm sido propostas para relacionar a propriocepção entre os idosos ${ }^{13}$ e podem gerar resultados conflitantes. Desta forma, o objetivo deste estudo foi sistematizar os achados que relacionam o exercício físico com a propriocepção em idosos, uma vez que ganhos proprioceptivos podem diminuir o risco de queda e consequentemente melhorar qualidade de vida entre os idosos.

\section{MÉTODOS}

Esta revisão foi realizada com pesquisa nas bases de dados Pubmed/Mediline, Lilacs e Scopus. As palavras-chaves utilizadas em combinação para busca de artigos foram: "elderly" e seu termo relacionado "aged", "proprioception" e termo relacionado "sense position" e "exercise", todos presentes como descritores em ciências da saúde (DeCS e Mesh). Para contemplar os três descritores e seus sinônimos na pesquisa, a seguinte associação de palavras foi realizada:

(elderly OR aged) AND (proprioception OR position sense) AND (exercise)

Para inclusão dos estudos uma série de critérios foi estabelecida e encontra-se no quadro 1.

Após junção do resultado das três bases de dados e retirada dos artigos repetidos, a busca delimitou artigos publicados na última década que demonstravam tratar especificamente sobre o assunto, uma vez que continham os descritores no título, resumo ou palavras chave. Posteriormente a estas delimitações, foi realizada uma análise independente e encontros de consenso entre dois autores do manuscrito para inclusão dos artigos que atendessem todos os critérios estabelecidos. 
QUADRO 1 - Lista de critérios para inclusão de estudos.

\begin{tabular}{l} 
Conter os descritores no título, resumo ou palavras-chave \\
\hline Publicados nos últimos 10 anos \\
\hline Apresentar mensuração da propriocepção \\
\hline Não direcionados a grupos com patologias ou deficiências específicas \\
\hline Artigos originais \\
\hline Disponíveis na língua inglesa \\
\hline Grupos organizados com número superior a 10 sujeitos \\
\hline Conter grupo com sujeitos acima de 60 anos \\
\hline Disponíveis até 31 de dezembro de 2013
\end{tabular}

Os estudos relevantes foram selecionados a partir da análise dos resumos, quando as informações eram insuficientes, o texto era lido na integra. No fim da avaliação, 9 artigos, que cumpriram os critérios de inclusão e atenderam os objetivos do estudo, foram utilizados para compor esta revisão sistemática.

\section{RESULTADOS}

A busca realizada nas três bases de dados resultou em 271 artigos encontrados. Após a verificação dos critérios propostos, 9 artigos foram selecionados para a revisão sistemática. (Figura 1).

Entre os artigos selecionados, dois consistem em um estudo transversal ${ }^{13}$, ${ }^{14}$, um estudo quase-experimental ${ }^{15}$ e seis estudos experimentais ${ }^{16-21}$ sendo que um deles teve seguimento (follow up) ${ }^{18}$.

Todos os artigos mostram uma associação com algum exercício, entre eles, foram encontradas modalidades como: Thai $\mathrm{Chi}^{13,14,17}$, Golfe ${ }^{14}$, natação, corrida ${ }^{13}$, fortalecimento muscular com ou sem auxilio de pesos livres, flexibilidade, caminhada ${ }^{16,18-20}$ e outros exercícios de agilidade ${ }^{21}$, equilíbrio ${ }^{18,20}$, postura e a propriocepção ${ }^{15-17,19,21}$.

Entre os componentes dos programas houve predominância de treinamento multifatorial, que combinavam exercícios resistidos, com flexibilidade, exercícios cardiovasculares e equilíbrio. Exercícios de propriocepção foram vistos em dois programas de treinamento dos nove artigos avaliados, sendo que entre um deles o treino proprioceptivo era base do programa ${ }^{17}$ e para o outro, combinava a prática da sensação articular com outros exercícios ${ }^{16}$.

Todos os artigos relacionados avaliaram propriocepção. Três estudos ${ }^{13-15}$ apresentaram como objetivo verificar a relação entre exercício e propriocepção e os outros seis estudos ${ }^{16-21}$ usaram a propriocepção como uma variável para verificar a relação entre o exercício e a funcionalidade do idoso e/ou o risco de quedas na terceira idade.

A propriocepção foi testada com o alinhamento dos membros inferiores ${ }^{18-21}$ a partir de uma placa de acrílico, que continha medidas divididas em graus, sendo que os sujeitos deveriam alinhar o membro dominante com o outro membro. Outro método utilizado para verificar a propriocepção foi a reposição passiva dos membros inferiores, no qual os participantes, posicionados em um aparelho específico, deveriam avisar assim que notassem o início do movimento e sua direção ${ }^{13}$, ou quando o membro em questão atingisse uma angulação igual a posição determinada previamente ${ }^{14,16,17}$. Ju et al. ${ }^{15}$, também avaliaram a propriocepção com o método de reposicionamento do membro, porém a posição de teste foi atingida de forma passiva, enquanto o reposi- 
cionamento do membro deveria ser feito de forma ativa. $\mathrm{O}$ desempenho dos avaliados foi medido através do erro da posição do membro, em graus.

Entre os resultados foram observados que o grupo idoso tem um erro maior da propriocepção comparado ao grupo jovem ${ }^{15}$. A melhora da propriocepção foi observada após intervenção multifatorial (fortalecimento, alongamento, exercícios aeróbios, proprioceptivos e cognitivos) ${ }^{16}$, multifatorial supervisionado ${ }^{20}$, com aulas de Thai $\mathrm{Chi}^{13,17}$ e exercícios de propriocepção ${ }^{17}$. Golfistas demonstraram níveis proprioceptivos melhores que sedentários e equivalentes aos praticantes de Tai chi chuan ${ }^{14}$. Lee et al. ${ }^{18}$ apresentaram um resultado conflitante de melhora na propriocepção apenas para o grupo controle. Enquanto de Carvalho Bastone et al. ${ }^{19}$ não verificaram melhora em nenhum grupo após o período de treinamento com exercícios multifatorial. Por fim, treinamento isolado de flexibilidade, agilidade e treinamento resistido não foi suficiente para promover modificações sobre a propriocepção ${ }^{21}$.

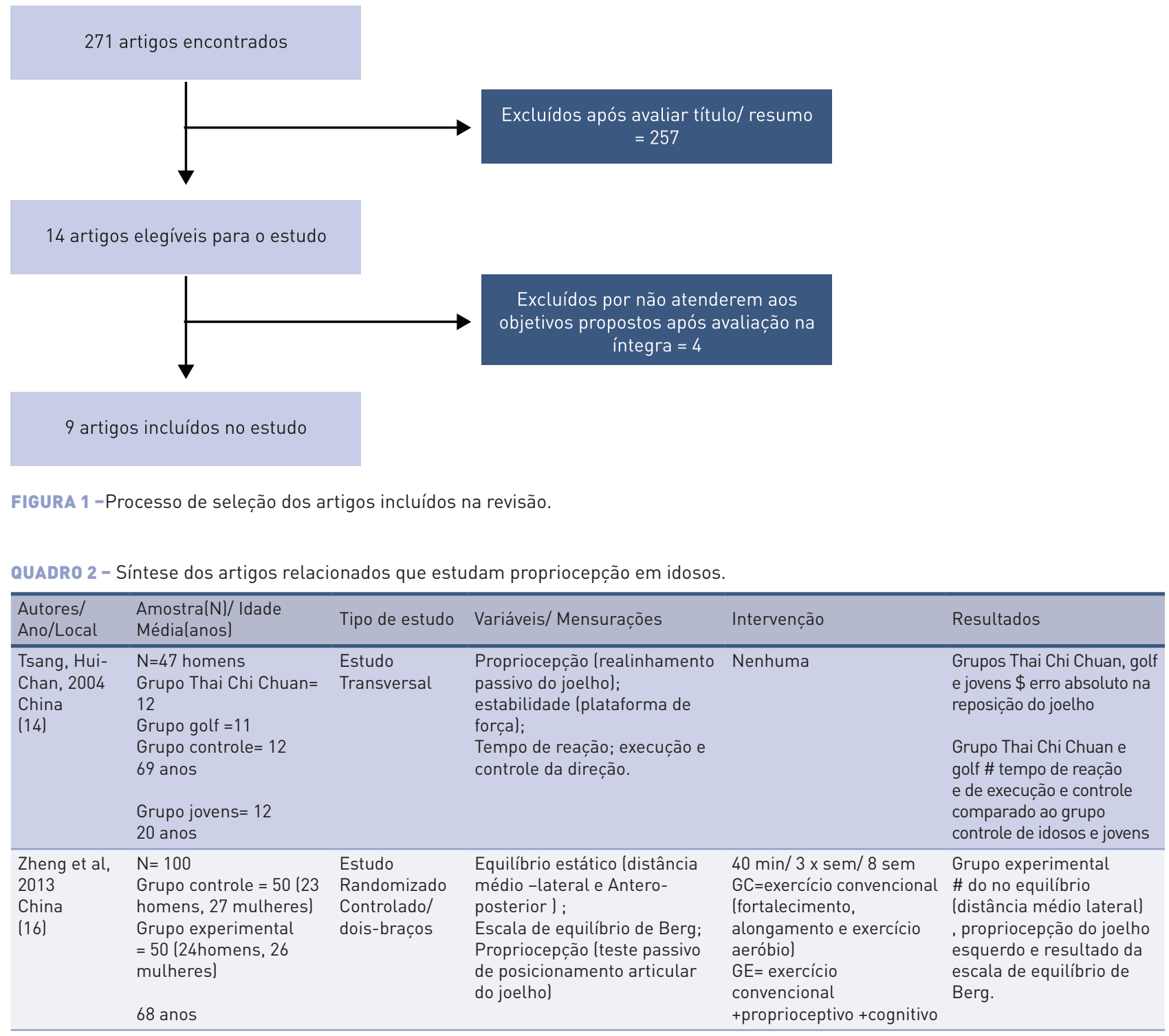




\begin{tabular}{|c|c|c|c|c|c|}
\hline $\begin{array}{l}\text { Autores/ } \\
\text { Ano/Local }\end{array}$ & $\begin{array}{l}\text { Amostra(N)/ Idade } \\
\text { Médialanos) }\end{array}$ & Tipo de estudo & Variáveis/ Mensurações & Intervenção & Resultados \\
\hline $\begin{array}{l}\text { Liu et al, } \\
2012 \\
\text { China } \\
\text { (17) }\end{array}$ & $\begin{array}{l}\text { N=42 } \\
\text { Grupo Thai Chi } \\
\text { Chuan=15 ( } 7 \text { homens, } 8 \\
\text { mulheres) } \\
\text { Grupo propriocepção= } \\
10 \text { ( } 4 \text { homens, } 6 \\
\text { mulheres) } \\
\text { Grupo controle= } 17 \text { ( } 7 \\
\text { homens, } 10 \text { mulheres) } \\
68 \text { anos }\end{array}$ & $\begin{array}{l}\text { Estudo } \\
\text { Randomizado } \\
\text { Controlado }\end{array}$ & $\begin{array}{l}\text { Propriocepção (teste passivo } \\
\text { de posicionamento articular } \\
\text { do tornozelo); } \\
\text { Força muscular (isocinético } \\
\text { - pico de torque de flexão e } \\
\text { dorsiflexão de tornozelo) }\end{array}$ & $\begin{array}{l}45 \text { min/ 2x sem / } 16 \text { sem } \\
\text { Grupo Thai Chi Chuan = } \\
\text { aula de Thai chi Chuan } \\
\text { Grupo Propriocepção= } \\
\text { exercícios proprioceptivos } \\
\text { Grupo controle= sem } \\
\text { exercícios }\end{array}$ & $\begin{array}{l}\text { \# propriocepção no } \\
\text { Grupo Thai Chi Chuan e } \\
\text { Grupo Propriocepção em } \\
\text { comparação ao Grupo } \\
\text { controle, mas não entre } \\
\text { grupo Thai Chi Chuan } \\
\text { e grupo propriocepção; } \\
\text { sem diferença na força. }\end{array}$ \\
\hline $\begin{array}{l}\text { Lee et al, } \\
2013 \\
\text { Taiwan (18) }\end{array}$ & $\begin{array}{l}\mathrm{N}=616 \\
\mathrm{~N}=616 \\
\text { Grupo controle }=303 \\
\text { (133 homens, } 170 \\
\text { mulheres) } \\
\text { Grupo experimental = } \\
313 \text { (147 homens, } 166 \\
\text { mulheres) } \\
76 \text { anos }\end{array}$ & $\begin{array}{l}\text { Estudo } \\
\text { Randomizado } \\
\text { Controlado } \\
\text { Follow up }\end{array}$ & $\begin{array}{l}\text { Nível de atividade física, } \\
\text { (IPAQ), nível cognitivo (Mini } \\
\text { mental), depressão, TUG, } \\
\text { risco de quedas, PPA } \\
\text { - (sensibilidade visual, } \\
\text { propriocepção, força dos MMII, } \\
\text { tempo de reação, teste de } \\
\text { controle postural) }\end{array}$ & $\begin{array}{l}50 \text { a } 60 \text { min / } 1 \text { x sem/ } \\
8 \text { sem } \\
\text { Exercícios acompanhados } \\
\text { + exercícios em casa } \\
\text { (total } 3 \text { meses) } \\
\text { GE= equilíbrio, } \\
\text { alongamento, resistência } \\
\text { cardiorrespiratória e } \\
\text { fortalecimento muscular, } \\
\text { aulas de risco e } \\
\text { prevenção de quedas e } \\
\text { perigos domésticos. } \\
\text { GC= receberam cadernos } \\
\text { sobre educação da saúde. } \\
12 \text { meses - seguindo } \\
\text { (risco de quedas) }\end{array}$ & $\begin{array}{l}\text { Grupo experimental \# } \\
\text { comparado ao grupo } \\
\text { controle no tempo de } \\
\text { reação, controle postural } \\
\text { com olhos abertos, teste } \\
\text { TUG e nível de depressão. } \\
\text { Grupo controle \# na força } \\
\text { de flexores de joelhos e } \\
\text { propriocepção. }\end{array}$ \\
\hline $\begin{array}{l}\text { De Carvalho } \\
\text { Bastone, } \\
\text { 2004/ } \\
\text { Brasil } \\
\text { (19) }\end{array}$ & $\begin{array}{l}\mathrm{N}=37 \\
\text { Grupo controle }=18 \text { ( } 4 \\
\text { homens, } 14 \text { mulheres) } \\
\text { Grupo experimental } \\
=19 \text { ( } 5 \text { homens, } 14 \\
\text { mulheres) } \\
78 \text { anos }\end{array}$ & $\begin{array}{l}\text { Estudo } \\
\text { Randomizado } \\
\text { Controlado }\end{array}$ & $\begin{array}{l}\text { Funcionalidade (curso com } \\
\text { obstáculos, velocidade da } \\
\text { marcha e função do MMII); } \\
\text { Força (extensores de joelho), } \\
\text { propriocepção, depressão e } \\
\text { cognição. }\end{array}$ & $\begin{array}{l}1 \mathrm{~h} / 2 \text { x sem / } 6 \text { meses. } \\
\text { GE = exercícios } \\
\text { de mobilidade, } \\
\text { fortalecimento, } \\
\text { caminhada e } \\
\text { relaxamento. } \\
\text { GC = sem exercícios }\end{array}$ & $\begin{array}{l}\text { Grupo experimental \# } \\
\text { nos testes funcionais, } \\
\text { funcionalidade do } \\
\text { MMII, velocidade da } \\
\text { marcha, força e \$ nível } \\
\text { de depressão. Não } \\
\text { houve diferença na } \\
\text { propriocepção. }\end{array}$ \\
\hline $\begin{array}{l}\text { Ju et al, } 2013 \\
\text { Taiwan } \\
\text { (15) }\end{array}$ & $\begin{array}{l}\mathrm{N}=24 \\
\text { Grupo jovem = } 12 \text { ( } 8 \\
\text { homens, } 4 \text { mulheres) } \\
24 \text { anos } \\
\text { Grupo idoso = } 12 \text { (3 } \\
\text { homens, } 9 \text { mulheres) } \\
72 \text { anos }\end{array}$ & $\begin{array}{l}\text { Estudo Quase } \\
\text { Experimental }\end{array}$ & $\begin{array}{l}\text { Propriocepção (teste ativo de } \\
\text { reposicionamento articular } \\
\text { do joelho e teste de cinestesia } \\
\text { articular). }\end{array}$ & $\begin{array}{l}60 \text { repetições / } 2 \text { dias } \\
\text { Movimentos passivos } \\
\text { repetitivos } \\
2 \text { máquinas (isocinético/ } \\
\text { design próprio) }\end{array}$ & $\begin{array}{l}\text { Grupo idosos \# erro e } \\
\text { variabilidade na reposição } \\
\text { articular, e sensação } \\
\text { de movimento passivo, } \\
\text { comparado ao grupo } \\
\text { jovem. } \\
\text { Sem diferenças entre } \\
\text { máquinas. }\end{array}$ \\
\hline $\begin{array}{l}\text { Donat, } 2007 \\
\text { Turquia } \\
\text { (20) }\end{array}$ & $\begin{array}{l}=32 \\
\text { Grupo sem supervisão } \\
=15 \text { (5 homens, } 10 \\
\text { mulheres) } \\
\text { Grupo com supervisão } \\
=17 \text { ( } 7 \text { homens, } 10 \\
\text { mulheres) } \\
80 \text { anos }\end{array}$ & $\begin{array}{l}\text { Estudo } \\
\text { Randomizado } \\
\text { Controlado }\end{array}$ & $\begin{array}{l}\text { Força de MMII (dinamômetro), } \\
\text { flexibilidade (sentar e } \\
\text { alcançar), medo de queda } \\
\text { (escala visual), funcionalidade } \\
\text { (TUG), equilíbrio (escala de } \\
\text { Berg) e propriocepção (senso } \\
\text { de posição da articulação do } \\
\text { joelho - igualar alinhamento } \\
\text { dos membros) }\end{array}$ & $\begin{array}{l}\text { 45-50 min / } 3 x \text { sem/ } 8 \text { sem } \\
\text { Exercícios de equilíbrio, } \\
\text { fortalecimento, posturais } \\
\text { e flexibilidade. } \\
\text { Grupo sem supervisão - } \\
\text { recebia as instruções e } \\
\text { deveria realizar os treinos } \\
\text { sem supervisão } \\
\text { Grupo com supervisão- } \\
\text { uma profissional } \\
\text { acompanhava os treinos. }\end{array}$ & $\begin{array}{l}\text { Ambos os grupos \# } \\
\text { equilíbrio (tempo em } \\
\text { tandem e em uma perna), } \\
\text { funcionalidade, (teste de } \\
\text { Berg), flexibilidade / } \\
\text { \# grupo com supervisão - } \\
\text { força e propriocepção. }\end{array}$ \\
\hline $\begin{array}{l}\text { Liu- } \\
\text { Ambrose, } \\
2004 \\
\text { Australia/ } \\
\text { Canadá } \\
\text { (21) }\end{array}$ & $\begin{array}{l}\text { N=98 mulheres } \\
\text { Grupo resistência }=32 \\
\text { Grupo Agilidade }=34 \\
\text { Grupo Flexibilidade }=32 \\
79 \text { anos }\end{array}$ & $\begin{array}{l}\text { Estudo } \\
\text { Randomizado } \\
\text { Controlado }\end{array}$ & $\begin{array}{l}\text { Questionário de saúde geral, } \\
\text { cognição e nível de atividade } \\
\text { física; medidas fisiológicas } \\
\text { (PPA - sensibilidade visual, } \\
\text { propriocepção, força dos MMII, } \\
\text { tempo de reação, teste de } \\
\text { controle postural). }\end{array}$ & $\begin{array}{l}50 \text { min / } 2 \text { x sem / } 25 \text { sem } \\
\text { Grupo resistência= } \\
\text { exercício com resistência, } \\
\text { intensidade } 75-85 \% \text {-1 } \\
\text { RM, } 2 \text { x- } 6 \text { - } 8 \text { rep. } \\
\text { G agilidade= jogos com } \\
\text { bola, dança, corrida com } \\
\text { obstáculo. } \\
\text { Grupo flexibilidade= } \\
\text { exercícios de } \\
\text { alongamento, relaxamento } \\
\text { e educação postural. }\end{array}$ & $\begin{array}{l}\text { Grupo agilidade \# escala } \\
\text { de mobilidade e equilíbrio } \\
\text { comparado aos outros } \\
\text { dois grupos. } \\
\text { Grupo resistência e } \\
\text { Grupo agilidade } \$ \text { risco } \\
\text { de quedas e melhor } \\
\text { estabilidade postural }\end{array}$ \\
\hline
\end{tabular}




\begin{tabular}{|c|c|c|c|c|c|}
\hline $\begin{array}{l}\text { Autores/ } \\
\text { Ano/Local }\end{array}$ & $\begin{array}{l}\text { Amostra(N)/ Idade } \\
\text { Média(anos) }\end{array}$ & Tipo de estudo & Variáveis/Mensurações & Intervenção & Resultados \\
\hline $\begin{array}{l}\text { Xu et al, } \\
2004 \\
\text { China } \\
\text { (13) }\end{array}$ & $\begin{array}{l}\mathrm{N}=68 \\
\text { Grupo Thai Chi Chuan } \\
=21(12 \text { homens, } 9 \\
\text { mulheres) } \\
\text { Grupo corrida ou } \\
\text { natação = } 20 \text { (12 } \\
\text { homens, } 8 \text { mulheres) } \\
\text { Grupo sem exercício } \\
=27 \text { (15 homens, } 12 \\
\text { mulheres) }\end{array}$ & $\begin{array}{l}\text { Estudo } \\
\text { Transversal }\end{array}$ & $\begin{array}{l}\text { Propriocepção do joelho e do } \\
\text { tornozelo (teste de cinestesia } \\
\text { articular) }\end{array}$ & Nenhuma & $\begin{array}{l}\text { Grupo Thai Chi Chuan } \\
\text { \# propriocepção de } \\
\text { tornozelo e na flexão de } \\
\text { joelho comparado ao } \\
\text { Grupo corrida ou natação } \\
\text { e Grupo controle. }\end{array}$ \\
\hline & 65 anos & & & & \\
\hline
\end{tabular}

TUG - time up and go / MMII - membro inferior / RM - repetição máxima / PPA - Protocolo de medidas fisiológicas.

\section{DISCUSSÃO}

O estudo teve como objetivo verificar associações existentes entre diferentes tipos de exercício físico e a propriocepção em idosos, visto que informações sensoriais influenciam o controle postural ${ }^{8,9}$ e consequentemente risco de quedas.

Exercício físico está relacionado com a melhora da propriocepção entre idosos ${ }^{11,12}$, todavia existe uma diversidade de indicações quanto ao tipo da atividade e frequência de prática que podem influenciar nos parâmetros proprioceptivos. Entre os exercícios encontrados são destacados os sessões de exercício multifatoriais ${ }^{16,18-20}$ e o Tai chi chuan ${ }^{13,14,17}$, além de outros programas alternativos que apareceram com menor frequência como o golfe $e^{14}$, natação/ corrida $^{13}$ e exercício de agilidade ${ }^{21}$.

\section{Programa de exercícios multifatoriais}

Os estudos que utilizaram um programa multifatorial de treinamento tiveram em comum a inclusão de exercícios de fortalecimento, alongamento ${ }^{20}$ e atividades aeróbias ${ }^{16,18,19}$. Além disso, exercícios de equilíbrio ${ }^{18,20}$ e de propriocepção ${ }^{16}$ também foram inseridos nos programas de intervenção.

Donat et al. ${ }^{20}$ mostraram melhoras significativas sobre a propriocepção no grupo que treinou com supervisão três vezes durante a semana. Zheng et al. ${ }^{16}$ também encontraram resultados positivos para o grupo que treinou com a mesma frequência de três vezes por semana. Porem a melhora da propriocepção foi observada apenas no grupo que participou de um programa convencional (fortalecimento, alongamento e aeróbio) conjugado com exercícios específicos de propriocepção. Por outro lado, de Carvalho Bastone et al. ${ }^{19}$ não apresentaram melhoras na propriocepção, sugerindo que uma intervenção convencional não seria suficiente para ter efeito sobre o sistema sensorial periférico ${ }^{19}$.

Lee et al. ${ }^{18}$ apresentaram um resultado conflitante, em que o grupo controle obteve melhoras na propriocepção, enquanto o grupo experimental não melhorou. A diferença entre o nível proprioceptivo inicial do estudo pode sugerir este resultado.

Uma limitação dos estudos multifatoriais se relaciona com a falta de dados sobre a contribuição isolada de cada exercício para a sensação articular. 
Com os resultados descritos acima, pode-se sugerir que programas multifatoriais que contenham exercícios específicos de propriocepção ou equilíbrio, realizados pelo menos três vezes por semana com algum tipo de supervisão, podem ser eficazes para desenvolver a propriocepção em idosos.

\section{Tai chi chuan}

A prática do Tai chi chuan tem sido sugerida como uma das atividades que melhoram a propriocepção pela relação que seu treinamento possui com o aumento da sensação consciente da posição do corpo ${ }^{4}$.

A prática do Tai chi chuan desafia o sistema a manter o equilíbrio ${ }^{14}$, requer movimentos contínuos e lentos, coordenados com diferentes amplitudes de desempenho ${ }^{17}$, mudanças de bases de apoio, movimentos uni e bilaterais, sempre enfatizando a consciência corporal e da realização dos exercícios ${ }^{13}$. Com isso esta modalidade pode ajudar a manter a sensibilidade de proprioceptores nas cápsulas articulares, ligamentos, tendões e músculos ${ }^{13}$.

Estudos demonstram que idosos praticantes de Tai chi chuan possuem melhor propriocepção que idosos sedentários ${ }^{13}{ }^{14,17}$, praticantes de natação e ou corrida $^{13}$, efeitos semelhantes são encontrados para treinamento específico de propriocepção ${ }^{17}$, praticantes de golfe e análogos de jovens para erro absoluto em teste reposicionamento articular do joelho ${ }^{14}$.

Outras vantagens somadas na escolha do Tai chi chuan como exercício para melhorar a propriocepção estão relacionados com a sua praticidade, já que esta modalidade não requer nenhum equipamento especial e pode ser realizada a qualquer momento e em qualquer lugar e com o alto nível motivacional, interesse e satisfação relatada por seus praticantes ${ }^{17}$.

\section{Programas alternativos}

Movimentos passivos repetitivos podem ser uma alternativa para melhorar a propriocepção ${ }^{15}$. Apesar de seu mecanismo não ser totalmente conhecido Ju et al. ${ }^{15}$, sugerem que a partir do ciclo de movimentos repetidos, a sensibilidade dos mecanoreceptores pode ser aumentada e a tensão muscular minimizada, melhorando a transmissão de informações dos movimentos articulares e por consequência a sensibilidade articular.

Idosos praticantes de natação e/ou corrida apresentaram melhor propriocepção que seus pares sedentários, porém resultados menos proeminentes do que idosos que praticam o Tai chi chuan ${ }^{13}$. Uma justificativa para estes resultados é que o exercício físico atenua declínios proprioceptivos relacionados com a idade, mas natação e corrida, que são atividades cíclicas, podem não exigir do sistema somatosensorial a mesma complexidade que outros exercícios como o Tai chi chuan demandam e por isto não obtêm resultados tão expressivos. Por outro lado, o Golfe é um exercício que exige concentração da mente, bom equilíbrio, coordenação de tronco e movimento de braços para tacada com precisão, assim o seu treino proporciona melhor capacidade proprioceptiva que a verificada em sedentários da mesma faixa etária e a mesma que idosos praticantes de Tai chi chuan ${ }^{14}$.

Outra possibilidade para atenuar o declínio da sensação consciente da posição do corpo decorrente do envelhecimento é através do treinamento específico da propriocepção, que revela efeitos positivos com menor erro sobre teste de percepção de posicionamento articular ${ }^{17}$. 
Atividades isoladas de agilidade e flexibilidade realizados duas vezes por semana, apesar de apresentarem efeitos sobre a estabilidade postural, não constataram modificações significativas para a propriocepção ${ }^{21}$. Treinamento resistido duas vezes por semana também não foi suficiente para aprimorar a sensação da posição $\operatorname{articular}^{21}$. A falta de associação com outras atividades na intervenção e a baixa frequência de treino podem ser possíveis explicações para estes resultados.

Os resultados do estudo sugerem que programas multifatoriais que contemplem exercícios específicos de propriocepção ou equilíbrio, realizados pelo menos três vezes por semana são eficazes para desenvolver a propriocepção em idosos.

Outras atividades como o Tai chi chuan que enfatiza a consciência corporal e contempla a capacidade de equilíbrio, coordenação e controle postural, o golfe que demanda concentração e exige bom domínio corporal para realizar a tacada com precisão, exercícios de movimentos passivos repetitivos e atividades específicas de propriocepção, proporcionam resultados positivos sobre a sensação consciente da posição do corpo. As modalidades de natação e corrida, por serem atividades cíclicas parecem ser menos eficientes nas melhorias proprioceptivas. Por outro lado, atividades isoladas de agilidade, flexibilidade e treinamento resistido, realizados duas vezes por semana, não demonstraram efeitos significativo sobre a propriocepção.

\section{Conclusão}

Considerando as diferentes propostas de exercício físico para desenvolvimento da propriocepção entre os idosos, Tai chi chuan, séries multifatoriais, golfe e exercícios de propriocepção demonstraram ser as estratégias mais espressivas. Todavia condução de estudos controlados randomizados com grande número de amostra se fazem necessários para confirmações conclusivas sobre os efeitos do exercício físico na propriocepção de idosos.

\section{REFERÊNCIAS}

1. Lowry KA, Vallejo AN, Studenski SA. Successful aging as a continuum of functional independence: lessons from physical disability models of aging. Aging Dis. 2012;3:5.

2. Bento PCB, Rodacki ALF, Homann D, Leite N. Exercícios físicos e redução de quedas em idosos: uma revisão sistemática. Rev Bras Cineantropom Desempenho Hum. 2010;12:471-9.

3. Franco PG, Rodacki ALF. Percepção de posicionamento articular e do nível de força em sujeitos idosos e jovens. Rev Edu Fís/UEM. 2011;22:327-35.

4. Goble DJ, Coxon JP, Wenderoth N, Van Impe A, Swinnen SP. Proprioceptive sensibility in the elderly: degeneration, functional consequences and plastic-adaptive processes. Neurosci Biobehav Rev. 2009;33:271-8.

5. Ahmed AF. Effect of sensorimotor training on balance in elderly patients with knee osteoarthritis. J Advanced Res. 2011;2:305-11.

6. Dijkerman HC, de Haan EH. Somatosensory processing subserving perception and action: Dissociations, interactions, and integration. Behav Brain Sci. 2007;30:224-30.

7. Lin S-I. Motor function and joint position sense in relation to gait performance in chronic stroke patients. Arch phys med rehabil. 2005;86:197-203.

8. Toledo DR, JA B. Diferenças sensoriais e motoras entre jovens e idosos: contribuição somatossensorial no controle postural. Rev Bras Fisioter. 2010;14:267-75.

9. Meyer PF, Oddsson LI, De Luca CJ. The role of plantar cutaneous sensation in unperturbed stance. Exp brain res. 2004;156:505-12. 
10. Martínez-Amat A, Hita-Contreras F, Lomas-Vega R, Caballero-Martínez I, Alvarez PJ, Martínez-López E. Effects of 12-week proprioception training program on postural stability, gait and balance in older adults:a controlled clinical trial. J strength cond res/ National Strength \& Conditioning Association. 2012.

11. Antes DL, Contreira AR, Katzer JI, Corazza ST. Propriocepção de joelho em jovens e idosas praticantes de exercícios físicos Knee proprioception in physically active young and elderly women. Fisioterapia pesquisa. 2009:306.

12. Martimbianco ALC, Polachini LO, Chamlian TR, Masiero D. Efeitos da propriocepção no processo de reabilitação das fraturas de quadril:[revisão]; Effects of proprioception in the rehabilitation process of hip fractures:[review]. Acta ortop bras. 2008;16:112-6.

13. Xu D, Hong Y, Li J, Chan K. Effect of tai chi exercise on proprioception of ankle and knee joints in old people. Br J Sports Med. 2004;38:50-4.

14. Tsang WW, Hui-Chan CW. Effects of exercise on joint sense and balance in elderly men: Tai Chi versus golf. Med Sci Sports Exerc. 2004;36:658-67.

15. Ju Y-Y, Lin J-K, Cheng H-YK, Cheng C-H, Wong AM-K. Rapid repetitive passive movement promotes knee proprioception in the elderly. Eu Rev Aging Phys Act. 2013;10:133-9.

16. Zheng J, Pan Y, Hua Y, Shen H, Wang X, Zhang Y, et al. Strategic targeted exercise for preventing falls in elderly people. J Int Med Res. 2013;41:418-26.

17. Liu J, Wang X-Q, Zheng J-J, Pan Y-J, Hua Y-H, Zhao S-M, et al. Effects of Tai Chi versus Proprioception Exercise Program on Neuromuscular Function of the Ankle in Elderly People: A Randomized Controlled Trial. Evid Based Complement Alternat Med.2012.

18. Lee H-C, Chang K-C, Tsauo J-Y, Hung J-W, Huang Y-C, Lin S-I, et al. Effects of a Multifactorial Fall Prevention Program on Fall Incidence and Physical Function in Community-Dwelling Older Adults With Risk of Falls. Arch Phys Med Rehabil. 2013;94:606-15.

19. de Carvalho Bastone A, Filho W. Effect of an exercise program on functional performance of institutionalized elderly. J Rehabil Res Dev. 2004;41:659-68.

20. Donat H, Özcan A. Comparison of the effectiveness of two programmes on older adults at risk of falling: unsupervised home exercise and supervised group exercise. Clin Rehabil. 2007;21:273-83.

21. Liu-Ambrose T, Khan KM, Eng JJ, Janssen PA, Lord SR, Mckay HA. Resistance and Agility Training Reduce Fall Risk in Women Aged 75 to 85 with Low Bone Mass: A 6-Month Randomized, Controlled Trial. J Am Ger Soc. 2004;52:657-65.

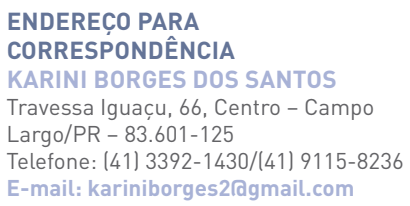

